\title{
Continuity of a class of entropies and relative entropies
}

\author{
Jan Naudts \\ Departement Natuurkunde, Universiteit Antwerpen, \\ Universiteitsplein 1, 2610 Antwerpen, Belgium \\ E-mail: Jan.Naudts@ua.ac.be
}

January 2004

\begin{abstract}
The present paper studies continuity of generalized entropy functions and relative entropies defined using the notion of a deformed logarithmic function. In particular, two distinct definitions of relative entropy are discussed. As an application, all considered entropies are shown to satisfy Lesche's stability condition. The entropies of Tsallis' nonextensive thermostatistics are taken as examples.
\end{abstract}

Keywords: Entropy, relative entropy, divergence, information content, Lesche's stability condition, generalized thermostatistics.

\section{Introduction}

The discrete entropy functional

$$
I_{0}(p)=-\sum_{k} p_{k} \log \left(p_{k}\right) \leq \infty
$$

is not continuous in the total variation norm

$$
\|p-q\|_{1}=\sum_{k}\left|p_{k}-q_{k}\right|
$$

in case the number of microstates $k$ is infinite. This means that a small change in probability distribution may cause an arbitrary large change in entropy. This discontinuity has been identified recently [1] as an essential 
characteristic of information content in natural languages. But its occurrence can make it difficult to obtain a reliable estimate of entropy from experimental observation. In many cases the probabilities $p_{k}$ are defined over a finite index set $k=1,2, \cdots, N$. Then uniform continuity holds and a useful estimate, called Lesche's stability condition [2], exists — see expression (55). The inequality was already known before since Fannes 3. proved the quantum version of the inequality about ten years earlier. However, Lesche formulated the inequality as a condition which is satisfied by (11) but not by the alpha-entropies of Rényi [4]. Recently [5], it has been shown that also the $q$-entropies of Tsallis' nonextensive thermostatistics satisfy Lesche's condition. Here, we generalize this proof to a large class of entropy functions, and formulate a more general continuity estimate (38).

It is known since long that in (11) the natural logarithm may be replaced by an arbitrary increasing function $f(x)$. The entropy of the discrete probability distribution function (pdf) $p$ reads then

$$
\tilde{I}(p)=-\sum_{k} p_{k} f\left(p_{k}\right)
$$

In the terminology of [ 6, 7, 8, these are quasi-entropies. It is clear that for general functions $f(x)$ not much can be said about continuity of entropy or relative entropy. It is obvious to require that $f(x)$ shares some of the properties of the natural logarithm. A class of functions satisfying such extra conditions has been introduced recently [9]. They have been used as the basis for a broad generalization of thermostatistics [10, 11]. The present paper focuses on entropy functionals occurring in this generalized thermostatistics.

A possible generalization of relative entropy, also called divergence [12], is $f$-divergence [13, 14, defined by

$$
I(p \| q)=\sum_{k} q_{k} f\left(p_{k} / q_{k}\right)
$$

with $f(x)$ a convex function, defined for $x>0$, strictly convex at $x=1$. The ratio $p_{k} / q_{k}$ can be seen as the discrete Radon-Nikodym derivative of $p$ w.r.t. $q$. The latter has been the basis for a systematic generalization to the context of quantum mechanics - see chapter 5 of [7]. Alternative expressions of the form

$$
D(p \| q)=\sum_{k}\left[f\left(p_{k}\right)-f\left(q_{k}\right)-\left(p_{k}-q_{k}\right) f^{\prime}\left(q_{k}\right)\right]
$$

with $f^{\prime}(x)$ the derivative of $f(x)$, are called divergences of the Bregman type in the mathematics literature. In the original definition [15] the pdfs $p$ and 
$q$ are interchanged. Then (4) and (5) are identical in case $f(x)=x \log (x)$. Hence, in the standard theory there is no need to make a difference between the two forms. To clarify why both are needed let us remark that mean entropy, in contrast with dynamical entropy, is negative relative entropy w.r.t. some reference state. If the number $N$ of microstates is finite then entropy is relative entropy w.r.t. uniform probabilities $q_{k}=1 / N$

$$
-I(p \| q)=-\frac{1}{N} \sum_{k} f\left(N p_{k}\right) .
$$

The continuum limit of (6) becomes

$$
-I(p \| q) \rightarrow-\int_{0}^{1} \mathrm{~d} k f(\rho(k))
$$

for any probability measure $p$ with density function $\rho(x)$ w.r.t. the Lebesgue measure $\mathrm{d} x$ of $[0,1]$. This continuum limit makes clear why a definition of relative entropy of the form (4) is needed. In what follows, the definition of generalized entropy that will be used is

$$
I(p)=-\sum_{k} f\left(p_{k}\right) .
$$

By omitting the factors $N$ from (6) the explicit dependence on the number of microstates disappears and the expression is of the form (3). In particular, if $f(x)=x \log (x)$ then $I(p)$ coincides with $I_{0}(p)$.

There exist also situations where a divergence of the form (5) is needed. In (generalized) statistical mechanics relative entropy $D(p \| q)$ measures the difference in free energy between an arbitrary pdf $p$ and the equilibrium pdf $q$. The quantity $-f^{\prime}\left(q_{k}\right)$ equals the energy of the $k$-the microstate divided by temperature (up to a constant term). Hence,

$$
-\sum_{k} p_{k} \ln _{\kappa}\left(q_{k}\right)-I(p)
$$

is the (non-equilibrium) free energy of $p$ divided by temperature $T$ (again up to a constant term). Then (5) expresses that free energy as a function of the $\operatorname{pdf} p$ is minimal at equilibrium $p=q$.

In information theory the linking identity connects average code length, entropy and divergence

$$
\langle\kappa, p\rangle=I(p)+D(p \| q) .
$$

See e.g. [1. Here, divergence measures the redundancy of the code $\kappa$ against the pdf $p$. From (10) follows

$$
\langle\kappa, p\rangle-\langle\kappa, q\rangle=I(p)+D(p \| q)-I(q),
$$


which can be identified with (5), provided that the average code length is given by

$$
\langle\kappa, p\rangle=-\sum_{k} p_{k} f^{\prime}\left(q_{k}\right)+C
$$

with $C$ a suitably chosen constant.

The paper is organized as follows. The next section gives a short review of deformed exponentials and logarithms. Sections 3, 4, and 5, discuss the definitions of entropy and relative entropy. Continuity estimates for entropy and relative entropy are given in section 6 . Finally, Lesche's stability condition is discussed in sections 7 and 8 . The paper is concluded with a short discussion of results, followed by appendices, containing proofs of inequalities.

\section{Deformed exponentials and logarithms}

In [9], a deformed logarithm is defined as a strictly increasing concave function, defined for all $x>0$, vanishing for $x=0$. Following [11] it is written as

$$
\ln _{\phi}(x)=\int_{1}^{x} \mathrm{~d} y \frac{1}{\phi(y)}
$$

with $\phi(y)$ a strictly positive increasing function. For convenience, the integral of $\ln _{\phi}(x)$ is denoted

$$
F_{\phi}(x)=\int_{1}^{x} \mathrm{~d} y \ln _{\phi}(y)=\int_{1}^{x} \mathrm{~d} y \frac{x-y}{\phi(y)} .
$$

The possible divergence of $\ln _{\phi}(x)$ at $x=0$ should be mild enough so that

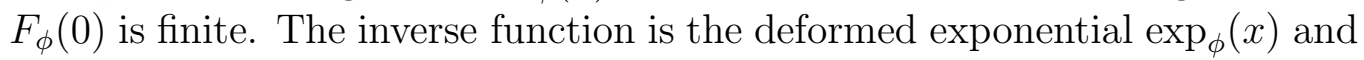
is defined on the range of $\ln _{\phi}(x)$, which may be less than the whole real line. If needed, the domain of definition is extended by putting $\exp _{\phi}(x)=0$ if $x$ is too small, and $\exp _{\phi}(x)=+\infty$ if $x$ is too large.

For further use the notion of deduced logarithmic function $\omega_{\phi}(x)$, associated with $\ln _{\phi}(x)$, is needed. It is defined by

$$
\begin{aligned}
\omega_{\phi}(x) & =(x-1) F_{\phi}(0)-x F_{\phi}(1 / x) \\
& =x \int_{0}^{1 / x} \mathrm{~d} y\left(-\ln _{\kappa}(y)-F_{\kappa}(0)\right) \\
& =\int_{0}^{1 / x} \mathrm{~d} y \frac{x y-1}{\phi(y)} .
\end{aligned}
$$

It is again a deformed logarithm provided that

$$
\int_{0}^{1} \mathrm{~d} x \ln _{\phi}(1 / x)<+\infty
$$


The name of $\kappa$-deformed logarithm is used in [9] and, with a more restricted meaning, in [16. To avoid confusion this name is used in the present paper only with the latter restricted meaning. Its origin is the kappa-distribution, which is a generalization of the Maxwell distribution. This distribution is given by

$$
\rho(v)=A\left[1+\frac{1}{2 \kappa} \beta \frac{v^{2}}{v_{0}^{2}}\right]^{-1-\kappa}
$$

and can be written as $\rho(v)=A \exp _{\phi}\left(-(1 / 2) \beta v^{2} / v_{0}^{2}\right)$ with the deformed logarithm $\ln _{\phi}(x)$ defined by

$$
\ln _{\phi}(x)=\kappa\left(1-x^{-1 /(1+\kappa)}\right)=\frac{\kappa}{1+\kappa} \int_{1}^{x} \mathrm{~d} y y^{-(2+\kappa) /(1+\kappa)}, \quad \kappa>0 .
$$

As a simple example of deformed exponential and logarithmic functions, consider the piecewise linear functions determined by the values

$$
\ln _{\phi}\left(a^{n}\right)=n, \quad \exp _{\phi}(n)=a^{n}, \quad n \in \mathbf{Z},
$$

with $a>0$ any base number. But also the function $\ln _{\phi}(x)=-1+\sqrt{x}$ is

a deformed logarithm. Its inverse is given by $\exp _{\phi}(x)=0$ if $x \leq-1$, and $\exp _{\phi}(x)=(1+x)^{2}$ otherwise.

\section{Entropy}

The entropy $I_{\phi}(p)$ of a discrete pdf $p$ is defined by means of the deduced logarithmic function $\omega_{\phi}(x)$, rather than by the deformed logarithm $\ln _{\phi}(x)$. The reason for doing so is that the derivative of $\omega_{\phi}(x)$ exists and can be calculated in terms of $\ln _{\phi}(x)$ while not much is known in general about the derivative $1 / \phi(x)$ of the function $\ln _{\phi}(x)$. The definition of entropy functional reads

$$
I_{\phi}(p)=\sum_{k} p_{k} \omega_{\phi}\left(1 / p_{k}\right) \leq+\infty .
$$

Note that the function $x \omega_{\phi}(1 / x)$ is non-negative and goes to zero in the limit $x=0$. Hence the expression is well-defined. Basic properties are $I_{\phi}(p) \geq 0$ and

$$
I_{\phi}(\lambda p+(1-\lambda) q) \geq \lambda I_{\phi}(p)+(1-\lambda) I_{\phi}(q), \quad 0 \leq \lambda \leq 1,
$$

i.e. entropy $I_{\phi}(p)$ is a concave function of the $\operatorname{pdf} p$.

From the definition of the deduced logarithmic function $\omega_{\phi}(x)$ follows that

$$
I_{\phi}(p)=\sum_{k}\left[\left(1-p_{k}\right) F_{\phi}(0)-F_{\phi}\left(p_{k}\right)\right]
$$




$$
=-F_{\phi}(0)-\sum_{k} \int_{0}^{p_{k}} \mathrm{~d} x \ln _{\phi}(x) .
$$

In particular, $I_{\phi}(p)$ is of the form (8) with

$$
f(x)=F_{\phi}(x)-(1-x) F_{\phi}(0) .
$$

Let us discuss some examples. If $\ln _{\phi}(x)$ is replaced by the natural logarithm $\log (x)$ then the entropy is denoted $I_{0}(p)$ and is given by the well-known expression (11). As a further example, consider entropy in the context of Tsallis' non-extensive thermodynamics [17. Fix a number $\kappa$ between -1 and 1 , not equal to 0 . A deformed logarithm is defined by

$$
\ln _{\phi}(x)=\left(1+\kappa^{-1}\right)\left(x^{\kappa}-1\right)=\int_{1}^{x} \mathrm{~d} y \frac{1+\kappa}{x^{1-\kappa}} .
$$

Note that this definition differs from the definition of $q$-logarithm found in the Tsallis literature [18, which coincides with the deduced logarithm

$$
\omega_{\phi}(x)=(1 / \kappa)\left(1-x^{-\kappa}\right)
$$

A short calculation yields the entropy functional

$$
I_{\phi}(p)=\frac{1}{\kappa}\left(1-\sum_{k} p_{k}^{1+\kappa}\right)
$$

This entropy functional was studied long ago by Havrda and Charvat [19] and by Daróczy [20]. It is a monotonic function of Rényi's alpha-entropies 4]. It is the starting point of Tsallis' thermostatistics. In the latter context it is common to use the parameter $q=1+\kappa$ instead of $\kappa$. In the present paper the symbols $p, q$, and $r$ are used for pdfs.

As a final example, consider the $\kappa$-deformed logarithm introduced by Kaniadakis [16, 21]

$$
\ln _{\kappa}(x)=\frac{1}{2 \kappa}\left(x^{\kappa}-x^{-\kappa}\right) .
$$

The parameter $\kappa$ should satisfy $-1<\kappa<1$ to guarantee concavity of the deformed logarithm. The inverse function reads

$$
\exp _{\kappa}(x)=\left(\kappa x+\sqrt{1+\kappa^{2} x^{2}}\right)^{1 / \kappa} .
$$

The corresponding entropy functional is obtained directly from (22). The result is

$$
I_{\kappa}(p)=\frac{1}{2 \kappa(1+\kappa)}\left(1-\sum_{k} p_{k}^{1+\kappa}\right)+\frac{1}{2 \kappa(1-\kappa)}\left(\sum_{k} p_{k}^{1-\kappa}-1\right) .
$$




\section{Relative entropy}

Let $q$ be a pdf for which $q_{k}>0$ holds for all $k$ (this condition can be omitted if the deformed logarithm is such that $\omega_{\phi}(0)$ is finite). From (4) follows that the relative entropy of the pdf $p$, given $q$, is defined by

$$
I_{\phi}(p \| q)=-\sum_{k} p_{k} \omega_{\phi}\left(q_{k} / p_{k}\right) .
$$

Note that, using the definition of $\omega_{\phi}$, one obtains

$$
I_{\phi}(p \| q)=\sum_{k} \int_{q_{k}}^{p_{k}} \mathrm{~d} x \ln _{\phi}\left(x / q_{k}\right)
$$

Expression (30) is of the form (44) with $f(x)$ given by (23). In particular, this means that the divergence $I_{\phi}(p \| q)$, considered here, is a special case of the $f$-divergence of [13, 14, with functions $f$ which are strictly convex and have a concave derivative. Many properties of $f$-divergence are known - see 22. In particular, one has $I_{\phi}(p \| q) \geq 0$ and $I_{\phi}(p \| q)=0$ implies $p=q$. Also, $I_{\phi}(p \| q)$ is jointly convex in $p$ and $q$.

For the example of Tsallis' entropy functional one obtains, using (25),

$$
I_{\phi}(p \| q)=\frac{1}{\kappa} \sum_{k} p_{k}\left(\left(\frac{p_{k}}{q_{k}}\right)^{\kappa}-1\right) .
$$

This expression has been introduced in the context of Tsallis' thermostatistics independently by several authors [23, 24, 25]. However, the definition was known before in the context of Rényi's alpha-entropies - see [26].

If $\ln _{\phi}(x)$ has a unique derivative $\ln _{\phi}^{\prime}(x)=1 / \phi(x)$ in the point $x=$ 1 and the probabilities $p_{k}$ depend on parameters $\theta^{i}$ then the generalized Fisher information metric [27], defined by $I_{\phi}(p+d p \| p)=I_{\phi}(p \| p+d p)=$ $(1 / 2) g_{i j}(p) \mathrm{d} \theta^{i} \mathrm{~d} \theta^{j}$, becomes

$$
g_{i j}(p)=\ln _{\kappa}^{\prime}(1) \sum_{k} p_{k} \frac{\partial \log \left(p_{k}\right)}{\partial \theta^{i}} \frac{\partial \log \left(p_{k}\right)}{\partial \theta^{j}} .
$$

Note that this expression does not depend on the actual choice of deformed logarithm, except through the prefactor $\ln _{\kappa}^{\prime}(1)$.

\section{Alternative definition of divergence}

So far, definition (30) seems quite satisfactory. However, as discussed in the introduction, there is a need for an alternative definition of the form (5) . By 
modification of (31) one obtains

$$
\begin{aligned}
D_{\phi}(p \| q) & =\sum_{k} \int_{q_{k}}^{p_{k}} \mathrm{~d} x\left(\ln _{\phi}(x)-\ln _{\phi}\left(q_{k}\right)\right) \\
& =\sum_{k}\left[F_{\phi}\left(p_{k}\right)-F_{\phi}\left(q_{k}\right)-\left(p_{k}-q_{k}\right) \ln _{\phi}\left(q_{k}\right)\right] \\
& =I_{\phi}(q)-I_{\phi}(p)-\sum_{k}\left(p_{k}-q_{k}\right) \ln _{\phi}\left(q_{k}\right) .
\end{aligned}
$$

This expression is of the form (51) with $f(x)$ given by (23). Positivity of $D_{\phi}(p \| q)$ follows immediately because $\ln _{\phi}(x)$ is an increasing function of $x$. Equality $D_{\phi}(p \| q)=0$ implies that $p=q$. Convexity in the first argument is straightforward. For the example of Tsallis' entropy one obtains

$$
D_{\phi}(p \| q)=\frac{1}{\kappa} \sum_{k} p_{k}\left(p_{k}^{\kappa}-q_{k}^{\kappa}\right)-\sum_{k}\left(p_{k}-q_{k}\right) q_{k}^{\kappa},
$$

which is definitely different from (32).

If the probabilities $p_{k}$ depend on parameters $\theta^{i}$ then the generalized Fisher information metric becomes

$$
g_{i j}(p)=\sum_{k} \ln _{\phi}^{\prime}\left(p_{k}\right) \frac{\partial p_{k}}{\partial \theta^{i}} \frac{\partial p_{k}}{\partial \theta^{j}} .
$$

Indeed, one has

$$
\begin{aligned}
D_{\phi}(p+\mathrm{d} p \| p) & =\sum_{k} \int_{p_{k}}^{p_{k}+\mathrm{d} p_{k}} \mathrm{~d} x\left(\ln _{\phi}(x)-\ln _{\phi}\left(p_{k}\right)\right) \\
& =\sum_{k} \int_{p_{k}}^{p_{k}+\mathrm{d} p_{k}} \mathrm{~d} x\left(\ln _{\phi}^{\prime}\left(p_{k}\right)\left(x-p_{k}\right)+\cdots\right) \\
& =\frac{1}{2} \sum_{k} \ln _{\phi}^{\prime}\left(p_{k}\right)\left(\mathrm{d} p_{k}\right)^{2}+\cdots,
\end{aligned}
$$

and similarly for $D_{\phi}(p \| p+\mathrm{d} p)$. In contrast with (33) the metric tensor (36) depends in a non-trivial way on the deformed logarithm $\ln _{\phi}$.

\section{Continuity estimates of entropy and of rel- ative entropy}

In Appendix $\mathrm{A}$ is proved that

$$
\left|I_{\phi}(p)-I_{\phi}(q)\right| \leq-\sum_{k} \int_{0}^{\left|p_{k}-q_{k}\right|} \ln _{\phi}(x) \mathrm{d} x
$$




$$
\begin{aligned}
& =\sum_{k}\left[F_{\phi}(0)-F_{\phi}\left(\left|p_{k}-q_{k}\right|\right)\right] \\
& \equiv d(p, q) \leq+\infty .
\end{aligned}
$$

The r.h.s. of (38) defines a metric $d(p, q)$. In particular, it satisfies the triangle inequality. Note that the distance between two pdfs may be infinite. This is not a problem since one can always define a new metric by $d_{M}(x, y)=$ $\min \{d(x, y), M\}$, with $M$ a fixed positive constant. The two metrics $d$ and $d_{M}$ define the same topology.

If $\ln _{\phi}(x)$ is taken to be the natural logarithm $\ln (x)$ then (38) becomes

$$
\left|I_{0}(p)-I_{0}(q)\right| \leq\|p-q\|_{1}-\sum_{k}\left|p_{k}-q_{k}\right| \ln \left(\left|p_{k}-q_{k}\right|\right) .
$$

More generally, take $\ln _{\phi}$ equal to the logarithm (24), used in the Tsallis context. Then (38) becomes

$$
\left|I_{\phi}(p)-I_{\phi}(q)\right| \leq\left(1+\kappa^{-1}\right)|| p-q \|_{1}-\kappa^{-1} \sum_{k}\left|p_{k}-q_{k}\right|^{1+\kappa} .
$$

Differences in relative entropy can be estimated in a way similar as for entropy differences. One finds (see Appendix A)

$$
\begin{aligned}
\left|I_{\phi}(p|| r)-I_{\phi}(q \| r)\right| & \leq d(p, q)+h_{r}(p, q) \\
\left|D_{\phi}(p \| r)-D_{\phi}(q \| r)\right| & \leq d(p, q)+e_{r}(p, q)
\end{aligned}
$$

with $d(p, q)$ as before, and with

$$
\begin{aligned}
& h_{r}(p, q)=\sum_{k}\left|p_{k}-q_{k}\right| \ln _{\phi}\left(1 / r_{k}\right), \\
& e_{r}(p, q)=-\sum_{k}\left|p_{k}-q_{k}\right| \ln _{\phi}\left(r_{k}\right)
\end{aligned}
$$

The r.h.s. of (41) is the sum of two distances, each satisfying the triangle inequality. Take $q=r$ in (41) to obtain an upper bound for $I_{\phi}(p \| q)$, resp. $D_{\phi}(p \| q)$.

\section{$7 \quad$ A general continuity condition}

The r.h.s. of (38) resembles the entropy of a distribution with elements $\mid p_{k}-$ $q_{k} \mid$. Introduce therefore the symmetric difference $p \Delta q$ of two distinct pdfs $p$ and $q$ by

$$
(p \Delta q)_{k}=\frac{\left|p_{k}-q_{k}\right|}{\|p-q\|_{1}}
$$


Note that $p \Delta q$ is again a pdf. Its elements satisfy $(p \Delta q)_{k} \leq 1 / 2$. This implies that

$$
I_{\phi}(p \Delta q) \geq-F_{\phi}(0)-\ln _{\phi}(1 / 2) .
$$

In Appendix B is shown that from (38) follows that if $\|p-q\|_{1} \leq 1$ then

$$
\left|I_{\phi}(p)-I_{\phi}(q)\right| \leq \frac{F_{\phi}(0)-F_{\phi}\left(\|p-q\|_{1}\right)}{F_{\phi}(0)}\left[F_{\phi}(0)+I_{\phi}(p \Delta q)\right] .
$$

If $\|p-q\|_{1}=1$, then this inequality coincides with (38).

Take $\ln _{\phi}$ equal to the logarithm (24), used in the Tsallis context. Then (45) becomes

$$
\left|I_{\phi}(p)-I_{\phi}(q)\right| \leq \frac{1}{\kappa}\left[(1+\kappa)\|p-q\|_{1}-\|p-q\|_{1}^{1+\kappa}\right]\left[1+I_{\phi}(p \Delta q)\right] .
$$

This is less sharp than (40) which can be written as

$$
\left|I_{\phi}(p)-I_{\phi}(q)\right| \leq \frac{1}{\kappa}(1+\kappa)\|p-q\|_{1}+\|p-q\|_{1}^{1+\kappa}\left[I_{\phi}(p \Delta q)-\frac{1}{\kappa}\right] .
$$

In combination with (44), (45) shows that the entropy functional $I_{\phi}(p)$ satisfies the following condition.

Condition 1 For each $\epsilon>0$ there exists $\delta>0$ such that

$$
|I(p)-I(q)| \leq \epsilon I(p \Delta q)
$$

holds for all $p d f s$ and $q$ satisfying $p \neq q$ and $\|p-q\|_{1} \leq \delta$.

To show relevance of this condition one consequence is highlighted. Note that $(\lambda p+(1-\lambda) q) \Delta q$ does not depend on $\lambda$ in the range $0<\lambda \leq 1$. Hence Condition 1 implies that for each $\epsilon>0$ there exists $\delta>0$ such that

$$
|I(\lambda p+(1-\lambda) q)-I(\mu p+(1-\mu) q)| \leq \epsilon I(p \Delta q)
$$

holds for distinct pairs $p$ and $q$, and for all $\lambda$ and $\mu$ between 0 and 1 , satisfying $|\lambda-\mu|\|p-q\|_{1} \leq \delta$. This result implies uniform continuity of entropy on the segment $(p, q)$, provided $I(p \Delta q)$ is finite. 


\section{Lesche's stability condition}

Assume now that the number of microstates is finite, equal to $N$ (i.e., the index $k$ of the pdfs $p$ and $q$ runs from 1 to $N)$. Introduce the notation

$$
I^{\max }(N)=\max \left\{I(p): p_{k}=0 \text { for } k>N\right\} .
$$

Lesche 2] showed twenty years ago that $I_{0}(p)$ satisfies the following condition.

Condition 2 For each $\epsilon>0$ there exists $\delta>0$ such that

$$
|I(p)-I(q)| \leq \epsilon I^{\max }(N)
$$

holds for all pdfs $p$ and $q$ satisfying $\|p-q\|_{1} \leq \delta$ and $p_{k}=q_{k}=0$ for $k>N$.

It is clear that an entropy function $I(p)$ satisfying Condition 1 also satisfies Condition 2. For fixed $N$ these conditions imply uniform continuity, which is a rather trivial statement because a continuous function on a compact set is automatically uniformly continuous. In addition, (51) specifies how the estimate depends on the number of nonzero components $N$.

In the remainder of this section some inequalities, used in the literature to prove Lesche's condition, are shown to follow from (38). In Appendix C is shown that (38) implies that

$$
\begin{aligned}
\left|I_{\phi}(p)-I_{\phi}(q)\right| & \leq N F_{\phi}(0)-N F_{\phi}\left(N^{-1}\|p-q\|_{1}\right) \\
& =-N \int_{0}^{\|p-q\|_{1} / N} \mathrm{~d} x \ln _{\phi}(x) \\
& =\|p-q\|_{1}\left[F_{\phi}(0)+\omega_{\phi}\left(N /\|p-q\|_{1}\right)\right] .
\end{aligned}
$$

It is difficult to bound $\omega_{\phi}\left(N /\|p-q\|_{1}\right)$ by $I_{\phi}^{\max }(N)=\omega_{\phi}(N)$ in the general case using only that $\omega_{\phi}(x)$ is a concave increasing function. However, in the case that the deformed logarithm is given by (24), then one has

$$
\omega_{\phi}\left(N /\|p-q\|_{1}\right)=\frac{1}{\kappa}\left(1-\|p-q\|_{1}^{\kappa}\right)+\|p-q\|_{1}^{\kappa} \omega_{\phi}(N) .
$$

This can be used to write (52) in the following form

$$
\begin{aligned}
\left|I_{\phi}(p)-I_{\phi}(q)\right| \leq & \left(1+\kappa^{-1}\right)\|p-q\|_{1} \\
& +\left[-\kappa^{-1}+I_{\phi}^{\max }(N)\right]\|p-q\|_{1}^{1+\kappa} .
\end{aligned}
$$

This is the result obtained recently by Abe [5]. It implies that $I_{\phi}(p)$ satisfies Condition 2. In the limit $\kappa=0$ (54) becomes

$$
\left|I_{0}(p)-I_{0}(q)\right| \leq\left(1+I_{0}^{\max }(N)\right)\|p-q\|_{1}-\|p-q\|_{1} \ln \left(\|p-q\|_{1}\right) .
$$

This is the expression obtained originally by Lesche [2]. Fannes [3, 28, showed that, if $\|p-q\|_{1} \leq 1 / 3$, then one has the slightly stronger inequality

$$
\left|I_{0}(p)-I_{0}(q)\right| \leq I_{0}^{\max }(N)\|p-q\|_{1}-\|p-q\|_{1} \ln \left(\|p-q\|_{1}\right) .
$$




\section{Discussion}

The present paper considers a large class of entropy functionals. Their definition is based on the concept of deformed logarithms. These entropies have nice enough properties to enable the proof of useful estimates. Only discrete pdfs have been considered. Expressions for continuous distributions and for quantum probabilities are found in [29].

For each entropy functional $I_{\phi}(p)$ there exists a metric $d(p, q)$ majorizing the difference $\left|I_{\phi}(p)-I_{\phi}(q)\right|$ - see inequality (38). The difference of relative entropies $\left|I_{\phi}(p \| r)-I_{\phi}(q \| r)\right|$ is majorized with the sum of two distances, the distance $d(p, q)$ mentioned above, and a distance $h_{r}(p, q)$ which depends on the pdf $r$ - see (41, 42).

An alternative definition of relative entropy $D_{\phi}(p \| q)$ has been proposed. It satisfies similar properties as $I_{\phi}(p \| q)$, but serves other goals. It is used in generalized statistical physics to measure changes in free energy. In information theory it is a measure of redundancy.

Although the proof of (38) is rather elementary, the result can be used to show that all entropy functionals, considered in the present paper, satisfy Lesche's stability condition (Condition 2 of the paper), as well as a stronger version of the inequality (Condition 1 of the paper). The proof is shorter and more transparent than that of $[5]$.

\section{Acknowledgement}

I thank Dr. P. Harremoës and Prof. H. Hasegawa for providing some of the references to the literature.

\section{Appendix A}

Here we prove the inequalities (38) and (41). Consider

$$
I_{\phi}(p)-I_{\phi}(q)=-\sum_{k} \int_{q_{k}}^{p_{k}} \mathrm{~d} x \ln _{\phi}(x) .
$$

If $p_{k}<q_{k}$ then the contribution is negative and may be omitted when trying to obtain an upperbound. Hence one gets immediately, using Heavisides function $\theta(x)$,

$$
I_{\phi}(p)-I_{\phi}(q) \leq-\sum_{k} \theta\left(p_{k}-q_{k}\right) \int_{0}^{p_{k}-q_{k}} \mathrm{~d} x \ln _{\phi}(x)
$$




$$
\leq-\sum_{k} \int_{0}^{\left|p_{k}-q_{k}\right|} \mathrm{d} x \ln _{\phi}(x)
$$

This proves (38).

To prove (41) note that from (31) follows

$$
I_{\phi}(p \| r)-I_{\phi}(q \| r)=\sum_{k} \int_{q_{k}}^{p_{k}} \mathrm{~d} x \ln _{\phi}\left(x / r_{k}\right) .
$$

Assume $p_{k}<q_{k}$ and write the $k$-th term as

$$
-\int_{p_{k}}^{q_{k}} \mathrm{~d} x \ln _{\phi}\left(x / r_{k}\right)
$$

It increases when $-\ln _{\phi}\left(x / r_{k}\right)$ is replaced by $-\ln _{\phi}(x)$. Hence the sum of all these terms is less than $d(p, q)$. On the other hand, if $p_{k} \geq q_{k}$ then the factor $\ln _{\phi}\left(x / r_{k}\right)$ in the $k$-th term can be replaced by $\ln _{\phi}\left(1 / r_{k}\right)$, which yields the bound

$$
\int_{q_{k}}^{p_{k}} \mathrm{~d} x \ln _{\phi}\left(x / r_{k}\right) \leq\left(p_{k}-q_{k}\right) \ln _{\phi}\left(1 / r_{k}\right) .
$$

The sum of these terms is bounded by $h_{r}(p, q)$. This finishes the proof of (41月).

In case of the alternative definition of divergence one has

$$
D_{\phi}(p \| r)-D_{\phi}(q \| r)=-I_{\phi}(p)+I_{\phi}(q)-\sum_{k}\left(p_{k}-q_{k}\right) \ln _{\phi}\left(r_{k}\right) .
$$

Hence, in this case the estimate is straightforward.

\section{Appendix B}

Here, expression (45) is derived. Note that any increasing concave function $g(x)$, satisfying $g(0) \geq 0$, also satisfies

$$
g(\lambda x) g(y) \leq g(x) g(\lambda y)
$$

for all $\lambda, x$, and $y$, for which $0 \leq \lambda \leq 1$ and $0<x<y$ hold. Apply this result with $g(x)=F_{\phi}(0)-F_{\phi}(x)$ (which is increasing on $\left.0 \leq x \leq 1\right), \lambda=\|p-q\|_{1}$, $x=\left|p_{k}-q_{k}\right| /\|p-q\|_{1}$, and $y=1$. Note that the assumption $\|p-q\|_{1} \leq 1$ is needed here. There follows, using $F_{\phi}(1)=0$,

$$
\begin{aligned}
& {\left[F_{\phi}(0)-F_{\phi}\left(\left|p_{k}-q_{k}\right|\right)\right] F_{\phi}(0)} \\
& \quad \leq\left[F_{\phi}(0)-F_{\phi}\left(\left|p_{k}-q_{k}\right| /\|p-q\|_{1}\right)\right]\left[F_{\phi}(0)-F_{\phi}\left(\|p-q\|_{1}\right)\right] .
\end{aligned}
$$

Using (38) this implies (45). 


\section{Appendix $\mathrm{C}$}

Here, inequality (52) is proved. Because $F_{\phi}(x)$ is convex one has for any $x$ and $a>0$

$$
F_{\phi}(x) \geq F_{\phi}(a)+(x-a) \ln _{\phi}(a) .
$$

Therefore (38) implies

$$
\left|I_{\phi}(p)-I_{\phi}(q)\right| \leq N F_{\phi}(0)-N F_{\phi}(a)-\left(\|p-q\|_{1}-N a\right) \ln _{\phi}(a) .
$$

The optimal choice of $a$ is $a=N^{-1}\|p-q\|_{1}$. This implies (52).

\section{References}

[1] P. Harremoës, F. Topsøe, Maximum entropy fundamentals, Entropy 3, 191-226 (2001).

[2] B. Lesche, Instabilities of Rényi entropies, J. Stat. Phys. 27, 419-423 (1982).

[3] M. Fannes, A continuity property of the energy density for spin lattice systems, Commun. math. phys. 31, 291-294 (1973).

[4] A. Rényi, On the foundations of information theory, Rev. Int. Stat. Inst. 33, 1-14 (1965).

[5] S. Abe, Stability of Tsallis entropy and instabilities of Rényi and normalized Tsallis entropies: A basis for q-exponential distributions, arXiv:cond-mat/0206078, Phys. Rev. E66, 046134 (2002).

[6] D. Petz, Quasi-entropies for finite quantum systems, Rep. Math. Phys. 23, 57-65 (1986).

[7] M. Ohya, D. Petz, Quantum entropy and its use (Springer-Verlag, 1993)

[8] D. Petz, Monotonicity of quantum relative entropy revisited, Rev. Math. Phys. 15(1), 79-91 (2003).

[9] J. Naudts, Deformed exponentials and logarithms in generalized thermostatistics, arXiv:cond-mat/0203489, Physica A, 316, 323-334 (2002).

[10] J. Naudts, Generalized thermostatistics and mean-field theory, arXiv:cond-mat/0211444, Physica A332, 279-300 (2004). 
[11] J. Naudts, Generalized thermostatistics based on deformed exponential and logarithmic functions, arXiv: cond-mat/0311438, to appear in Physica A.

[12] S. Kullback, R. Leibler On information and sufficiency, Ann. Math. Statist. 22, 79-86 (1951).

[13] I. Csiszár, Eine informationstheoretische Ungleichung und ihre Anwendung auf den Beweis der Ergodizität von Markoffschen Ketten, Maggar. Tud. Akad. Mat. KutatøInt. Közl. 8, 85-108 (1963).

[14] I. Csiszár, A class of measures of informativity of observation channels, Per. Math. Hung. 2(1-4), 191-213 (1972).

[15] L. M. Bregman, The relaxation method of finding a common point of convex sets and its application to the solution of problems in convex programming, USSR comp. math. math. phys., 7, 200-217 (1967).

[16] G. Kaniadakis and A.M. Scarfone, A new one parameter deformation of the exponential function, NEXT2001 Meeting, cond-mat/0109537, Physica A305, 69-75 (2002).

[17] C. Tsallis, Possible Generalization of Boltzmann-Gibbs Statistics, J. Stat. Phys. 52, 479-487 (1988).

[18] C. Tsallis, What are the numbers that experiments provide? Quimica Nova 17, 468 (1994)

[19] J. Havrda, F. Charvat, Kybernetica 3, 30-35 (1967).

[20] Z. Daróczy, Inform. Control 16, 36- (1970).

[21] G. Kaniadakis, Nonlinear kinetics underlying generalized statistics, Physica A296, 405-425 (2001).

[22] Inequalities for Csiszár f-Divergence in Information Theory, edited by S.S. Dragomir, RGMIA Monographs, Victoria University, 2000; ONLINE: http://rgmia.vu.edu.au/monographs/

[23] S. Abe, q-Deformed Entropies and Fisher Metrics, in: Proceedings of The 5th International Wigner Symposium, (August 25-29, 1997, Vienna, Austria), eds. P. Kasperkovitz and D. Grau (World Scientific, Singapore, 1998) p. 66.

[24] C. Tsallis, Generalized entropy-based criterion for consistent testing, Phys. Rev. E58(2), 1442-1445 (1998). 
[25] M. Shiino, H-theorem with generalized relative entropies and the Tsallis statistics, J. Phys. Soc. Jpn 67(11), 3658-3660 (1998).

[26] H. Hasegawa, $\alpha$-divergence of the non-commutative information geometry, Rep. Math. Phys. 33(1/2), 87-93 (1993).

[27] S. Amari, Differential-geometrical methods in statistics, Lecture Notes in Statistics 28 (1985).

[28] The quantum mechanical entropy of a state with density matrix $\rho$ is defined by $I_{0}(\rho)=-\operatorname{Tr} \rho \log \rho$. The inequality

$$
\left|I_{0}(\rho)-I_{0}(\sigma)\right| \leq \lambda \log N-\lambda \log \lambda
$$

holds with $\lambda=\operatorname{Tr}|\rho-\sigma|$ whenever $\lambda \leq 1 / 3$. Here, $N$ is the dimension of the Hilbert space, Tr is the trace. The inequality is quoted in [7], together with a proof, in Proposition 1.8, be it with quite different notations.

[29] J. Naudts, Non-unique way to generalize the Boltzmann-Gibbs distribution, arXiv:cond-mat/0303051. 